\title{
Optimizing optical systems with active components
}

Tibor Agócs

Lars Venema

Visa Korkiakoski

Gabby Kroes 


\title{
Optimizing optical systems with active components
}

\author{
Tibor Agócs*a, Lars Venema ${ }^{\mathrm{b}}$, Visa Korkiakoski ${ }^{\mathrm{c}}$, Gabby Kroes ${ }^{\mathrm{a}}$ \\ ${ }^{a}$ NOVA Optical Infrared Instrumentation Group at ASTRON, P.O. Box 2, 7990 AA Dwingeloo, The \\ Netherlands; \\ ${ }^{\mathrm{b}}$ ASTRON, P.O. Box 2, 7990 AA Dwingeloo, The Netherlands; \\ ${ }^{c}$ Leiden Observatory, Niels Bohrweg 2, NL-2333 CA Leiden, The Netherlands;
}

\begin{abstract}
The increasing requirement on the performance of optical instruments leads to more complex optical systems including active optical components. The role of these components is to correct for environmental influences on the instrument and reduce manufacturing and alignment residuals. We describe a method that can be used to design and operate instruments with active components that are not necessarily located in the pupil. After the optical system is designed, the next step is to analyse the available degrees of freedom (DOF), select the best set and include them in the active component. By performing singular value decomposition (SVD) and regularization of the sensitivity matrix, the most efficient DOF for the active component can be calculated. In operation of the instrument, the wavefront at the pupil plane is reconstructed from phase diversity (PD); a metrology having minimal impact on instrument design. Information from SVD, forward and reverse optimization are used to model the process, explore the parameter space and acquire knowledge on convergence. The results are presented for a specific problem.
\end{abstract}

Keywords: Active component, active instrument, phase diversity, optimization, reverse optimization, singular value decomposition

\section{INTRODUCTION}

The increasing requirements on the performance of optical systems lead to more complex optical systems and optical paths that should operate at accuracies comparable or better than that achieved in current instrumentation. For large scale structures like telescopes, the move towards active and even adaptive (active with high update frequencies) optics has been made, but at instrument level (excluding adaptive optics) this was up to recently not the case. However, also in more complex instruments, active optical paths become more and more common (cf. X-Shooter, VLTI).

An essential part of active systems is the metrology that should provide the necessary information to change the optical path to reach the desired performance. Having active optics in combination with good metrology system offers the advantage not only to provide the function for which it is included, but it can be used for the following points as well:

i. Reconfiguration of the instrument

ii. Removal of environmental influences on the instrument

iii. Reducing manufacturing residuals in optics

iv. Reducing alignment residuals from the optical train

The metrology in general is optimized for its application, which can be driven by different requirements. Continuous/incidental analysis, temporal and/or spatial resolution, level of added complexity to the optical path and level of non-commonality of the optics are criteria to take into account. We investigate the situation where the optical path is stable to a high degree and only incidental changes are required (either for determining residual path errors in existing active/adaptive systems or measuring slowly changing residual errors).

*agocs@astron.nl; phone +31 (0)52 15951 73; http://www.astron.nl/

Modern Technologies in Space- and Ground-based Telescopes and Instrumentation II, edited by Ramón Navarro, Colin R. Cunningham, Eric Prieto, Proc. of SPIE Vol. 8450, 84505F (C) 2012 SPIE · CCC code: 0277-786X/12/\$18 · doi: 10.1117/12.925326 
For this particular situation, there is a strong drive to use the science path including detector for monitoring the optical performance at the necessary spatial resolution. This prevents at the same time non-common path errors as well as it minimizes the changes needed in the optical system and does not require separate measurement devices. Phase diversity has been selected as method to derive the performance of the optical system. This work starts out using the simplest phase modification: defocus, where images are recorded and processed with an well-defined phase difference (defocus). The used phase retrieval technique is based on phase diversity and Gerchberg-Saxton algorithms and it is detailed in the paper by Korkiakoski et al [1]. Questions such as what accuracy can be achieved, how many phase diversions are needed and what is the convergence behaviour are addressed.

The phase retrieval technique estimates the wavefront phases in the pupil plane, directly coupled to the errors in the optical system, as the shape or position/orientation errors of optical components generate phase changes of the light. However, the impact of these errors on performance depends strongly on the location of the optical component in the system. The translation of the pupil phase to misalignments, production errors or mirror shape correction for components not in a pupil, is however another step to be taken, which is described in this report.

We used linear algebra techniques based on singular value decomposition and matrix truncation to relate the pupil aberration to the required changes on a particular surface. As will be described in the paper, a pre-selection of an independent set of adjustable parameters in the optical system (compensators or active components) is required to make the approach stable. Such compensators can be can be tilts, displacements or surface deformations.

In section 2, we present methods that translates available degrees of freedom of active components into wavefront errors in the pupil. Based on SVD and regularization of the sensitivity matrix we show how to select compensator DOF in the system. We also look at different optimization methods to minimize the performance metric wavefront phase or wavefront error (WFE). We use Newton's method and the SVD based pseudo-inverse of the sensitivity matrix to calculate compensator DOF adjustments iteratively. We also investigate reverse optimization, where the aim is to arrive to as close to the nominal configuration as possible. In the latter case we make use of the optimization algorithms of an optical ray-tracing program.

In section 3, we focus our work on compensating wavefront errors that were introduced due to two factors: misalignments and surface shape errors. We investigate the high-resolution spectrograph part of Metis (proposed E-ELT instrument), which is based on a double pass TMA (Three Mirror Anastigmat). Through SVD we study the optical system and its best possible DOF for misalignment compensation. We simulate alignment using the best set of compensators. In the second part we investigate the same double pass TMA with one component being an active mirror. We model the surface of the active mirror with orthogonal Zernike polynomials, find the correlation between the active mirror's surface and pupil WFE. We simulate degraded WFE and make corrections with the active mirror.

\section{SELECTION AND CONTROL OF ACTIVE COMPONENT}

Phase diversity provides the phase function (WFE) in the pupil of the system. Using this information, misalignments can be corrected and the system's performance improved. The correction or improvement can only be made efficiently when the coupling between the different degrees of freedom (DOF) of the system and their effect on the performance metric (WFE) is understood.

All available DOF define the configuration of an optical system and these DOF can be listed in the configuration vector. The optical performance of the system can be characterized by the system performance metric. The correlation between the configuration vector and the performance metric can be written:

$$
m=f(\boldsymbol{x})
$$

where $m$ is the performance metric, $\boldsymbol{x}$ is the configuration vector and $f$ is a function that defines their relation. Essentially we intend to find the best $\boldsymbol{x}$ that minimizes $m$. Usually, there is a maximum allowed $m_{\max }$, corresponding to the requirement of the system.

Usually $f$ is non-linear and there are interdependencies between the configuration parameters, which makes the inverse process (from the errors towards the change in configuration parameters) ill defined. We are following two steps to overcome these difficulties and minimize $m$. 
1. Through singular value decomposition of the sensitivity matrix we investigate the Eigen vectors of the optical system. The Eigen vectors define the best set of DOF that can be used as compensators. The number of DOF is limited to describe the majority of the variance in the optical system.

2. We are using optimization methods to minimize the performance metric and simulate the control of compensator DOF . We use Newton's method based on SVD and reverse optimization methods. The latter we implement in ray-tracing program Zemax ${ }^{\circledR}$ using its internal damped least squares (DLS) and orthogonal descent (OD) algorithms. We compare the optimization methods.

Our linear algebra methods are based on the ones presented by Chapman et al. [3] and Hvisc et al. [4].

\subsection{SVD and regularization of the sensitivity matrix}

The SVD of the sensitivity matrix is important to understand the sensitivities of the system and it can be used to get an insight to the type of aberration modes that the system can show due to misalignments. By regularizing the sensitivity matrix a subset of most efficient compensators could be defined.

The sensitivity matrix is based on the performance metric. The performance metric, in our case WFE, can be characterized by the aberration vector and its norm. The aberration vector can be defined in different ways. It can be formed by decomposing the WFE into $n$ Zernike polynomials $\left(z_{i}\right)$. The norm of the aberration vector is the RMS WFE, which is the performance metric of the system:

$$
m=\|\boldsymbol{z}\|=\sqrt{\sum_{i=1}^{n} z_{i}^{2}}
$$

Another way of defining the aberration vector is by sampling the exit pupil of the system and calculating the WFE at $n$ positions. The aberration vector components in this case consist of the WFE at every sampling position divided by $\sqrt{n}$. The norm of the vector gives the same result as above:

$$
m=\|\boldsymbol{z}\|=\sqrt{\frac{\sum_{i=1}^{n}\left(W F E_{i}\right)^{2}}{n}}
$$

The sensitivity matrix is defined as the Jacobian matrix evaluated about the nominal configuration:

$$
S_{i j}=\partial z_{i} / \partial x_{j}
$$

It can be calculated from the model of the system by dividing $\left(W F E_{\text {perturbed }, i}-W F E_{\text {nominal }, i}\right)$ or $\left(z_{\text {perturbed }, i}-\right.$ $\left.z_{\text {nominal }, i}\right)$ with the perturbation of $x_{j}$. In practice some unit perturbations are used and the sensitivity matrix is formed from the aberration vectors directly. Naturally in this case the sensitivity matrix depends on the defined units of perturbations.

To analyse the sensitivity matrix we use SVD, which can be given by the following formula:

$$
S=U \cdot W \cdot V^{T}
$$

where $\boldsymbol{U}$ and $\boldsymbol{V}$ are column orthonormal matrices and $\boldsymbol{W}$ is a diagonal matrix. Essentially, these matrices indicate the type of aberrations that result from changes in the DOF and they are listed from easiest to produce to hardest to produce. In its diagonal $W$ contains the singular values $\left(w_{1}, w_{2}\right.$, etc.), which measure the sensitivity of the system regarding a particular aberration mode. Also $w_{1} \geq w_{2} \geq \cdots$ and $w_{1}$ corresponds to the most sensitive aberration mode and it is followed by the other modes with decreasing sensitivity. In its columns $\boldsymbol{U}$ contains the aberration vector components for a given mode and $\boldsymbol{V}$ lists the changes in DOF that produced those aberrations. E.g. the most sensitive aberration mode is given by the first column vector from $\boldsymbol{U}$ that is resulted from the changes in DOF listed in the first column vector of $\boldsymbol{V}$ and has the strength of $w_{1}$.

By looking at the column vectors of $\boldsymbol{U}$ and $\boldsymbol{V}$ and the diagonal values of $\boldsymbol{W}$, it is possible to derive useful information on a given optical system. Usually not all DOF in a system are used for compensation, so it is worth to investigate how to select the best possible set of them. By regularizing the sensitivity matrix and constraining the number of the configuration vector components only to a best set, the optimization process will be stable and a converging solution can 
be found. The best set of compensators introduces aberrations that form the most linearly independent set within the sensitive subspace of aberrations. We are using SVD based subset selection detailed in [3] and [5]. As a result of the subset selection, the compensator DOF $\left(\boldsymbol{x}^{\prime}\right)$ are obtained and we can calculate the sensitivity matrix $S_{i j}^{\prime}=\partial z_{i} / \partial x_{j}^{\prime}$ and its SVD for these particular compensator DOF:

$$
S^{\prime}=U^{\prime} \cdot W^{\prime} \cdot V^{\prime T}
$$

While the original sensitivity matrix $S_{i j}$ includes all available DOF, $S^{\prime}{ }_{i j}$ consists only of the best compensator DOF and so it is a truncated sensitivity matrix. It can be determined experimentally, since the compensators are implemented as adjustable DOF in the system. The truncation of the sensitivity matrix results in a better conditioned matrix, which is also advantageous during the optimization (or alignment) process. Usually optimization at a certain stage involves linearization and matrix inversion is needed to solve a set of linear equations. By regularizing the sensitivity matrix, the number of singular values close to zero is significantly reduced and so the condition of the matrix is improved, which is essential to find a stable and converging solution.

As we intend to correct misalignments or improve performance metric in systems, which are relatively close to the nominal configuration this approach using the sensitivity matrix provides a good basis for the optimization process.

\subsection{Optimization algorithms}

In section 2.1, we presented linear algebra techniques that served to understand the possible aberrations caused by a small perturbation in different DOF and find the most efficient compensators of the system. Now we turn our attention towards the original problem of minimizing $\mathbf{z}(\boldsymbol{x})$ of an optical system that has misalignments or degraded image quality. With phase diversity, we can measure the aberration vector $\boldsymbol{z}_{\text {meas }}$ and we are looking for $\delta \boldsymbol{x}$, the necessary change in compensator DOF to minimize performance metric. Due to nonlinearity of $\boldsymbol{z}(\boldsymbol{x})$, we know that iterations are needed to find the best solution. In the following we define two different approaches: forward and reverse optimization methods. They have different performance metrics, the first minimizes overall WFE of the actual system, while the latter minimizes the difference between the nominal and actual WFE .

\subsubsection{Forward optimization}

The forward optimization method is independent of the nominal optical model. It uses the performance of the actual system and also the sensitivity matrix will be determined for the actual system. Then again the repetition of the SVD analysis determines for the actual system the most important DOF to improve the performance metric.

The performance metric in this case is the overall RMS WFE as it was defined before:

$$
m=\|\boldsymbol{z}(\boldsymbol{x})\|
$$

We use the Newton's method and SVD based pseudo-inverse calculation to find $\delta \mathbf{x}$ (more information on this can be found in [5]). In this method the aberration vector can be minimized by trying to find a solution to $z=0$. Using the Taylor expansion and neglecting all non-linear terms we arrive to the following:

$$
\begin{gathered}
0=\boldsymbol{z}(\boldsymbol{x}+\delta \boldsymbol{x})=\boldsymbol{z}(\boldsymbol{x})+\boldsymbol{J} \cdot \delta \boldsymbol{x} \\
\boldsymbol{J} \cdot \delta \boldsymbol{x}=-\boldsymbol{z}(\boldsymbol{x})
\end{gathered}
$$

where $J_{i j}=\partial z_{i} / \partial x_{j}$ is the Jacobian matrix. At this point we already know which are the most efficient compensators in our optical system and calculated or determined experimentally the sensitivity matrix, which we substitute for the Jacobian matrix:

$$
\boldsymbol{S}^{\prime} \cdot \delta \boldsymbol{x}=-\boldsymbol{z}(\boldsymbol{x})
$$

We arrived to a set of linear equations, which are overdetermined. We solve the linear equations in the least squares sense, calculate the pseudo-inverse of $\boldsymbol{S}^{\prime}$ with SVD and obtain the required change in compensator DOF:

$$
\delta \boldsymbol{x}=-\boldsymbol{V}^{\prime} \cdot \boldsymbol{W}^{\prime-1} \cdot \boldsymbol{U}^{\prime T} \cdot \mathbf{z}(\boldsymbol{x})
$$

In case the aberrations are larger the linear approach will not immediately converge to the optimal solution. Therefore, the process must be iterated during the alignment process. The wavefront is measured with phase diversity at every iteration $\left(\mathbf{z}_{\text {meas, }}\right)$ and we can define the following: 


$$
\begin{gathered}
\delta \boldsymbol{x}_{\boldsymbol{n}}=-\boldsymbol{V}^{\prime} \cdot \boldsymbol{W}^{\prime-1} \cdot \boldsymbol{U}^{\prime T} \cdot \boldsymbol{z}_{\text {meas }, n} \\
\boldsymbol{x}_{\boldsymbol{n}}=\boldsymbol{x}_{\boldsymbol{n}-\mathbf{1}}+\delta \boldsymbol{x}_{\boldsymbol{n}} \\
\boldsymbol{x}_{\boldsymbol{n}} \rightarrow \boldsymbol{z}_{\text {meas }, n+1}
\end{gathered}
$$

During alignment, the next configuration is calculated iteratively from the measured wavefront using the SVD of the truncated sensitivity matrix. In the ideal case the iteration continues until the performance metric reaches an acceptable value: $\boldsymbol{x}_{\text {final }}$.

In section 3, a particular optical system is analysed with the methods presented above. The calculation of the sensitivity matrix, the regularization and the optimization is done in Matlab ${ }^{\circledR}$ and Zemax ${ }^{\circledR}$. The communication between them is solved with Dynamic Data Exchange (DDE) [6].

\subsubsection{Reverse optimization}

A different way of finding $\delta \boldsymbol{x}$ from the $\mathbf{z}_{\text {meas }}$ aberration vector is with reverse optimization. In this case our purpose is to optimize the compensator DOF in order to arrive to the nominal configuration or as close to the nominal configuration as it is possible. We can define a new aberration vector and performance metric for the reverse optimization:

$$
m_{\text {rev }}=\left\|\mathbf{z}_{\text {rev }}\right\|=\left\|\mathbf{z}-\mathbf{z}_{\text {meas }}\right\|
$$

The optimization problem that we try to solve is to find the best compensator DOF values $\left(\delta \boldsymbol{x}_{\boldsymbol{f} \text { inal }}\right)$ that minimize the above defined metric. During alignment $\delta \boldsymbol{x}_{\text {final }}$ should be reversed in order to arrive to the nominal configuration: $\boldsymbol{x}_{\text {final }}=\boldsymbol{x}_{\mathbf{0}}-\delta \boldsymbol{x}_{\text {final }}$. For reverse optimization we use optical ray-tracing program Zemax ${ }^{\circledR}$ with its damped least squares (DLS) and the orthogonal descent (OD) algorithms. The advantage of using the ray-tracing program is that all iterations are done within its embedded algorithms and the calculated $\delta \boldsymbol{x}_{\text {final }}$ should be reversed at the end of the reverse optimization. Also here regularization is imperative to force the system to converge. The Jacobian matrix will not be calculated at the current position, but will be based on the nominal position. The convergence is at risk when the deviations are large and the linearized problem does not move the system in the right direction.

The reverse optimization method was implemented entirely by using the programming language of Zemax ${ }^{\circledR}$ and there was no need to use any additional programming languages. In the following we briefly present the two optimization methods that are embedded in Zemax®.

The orthogonal descent algorithm is similar to the Newton method presented before: negative of the gradient at a given configuration is used to calculate the next iteration. Then the inverse of the gradient is calculated in the least squares sense:

$$
\delta x=-\left(J^{T} \cdot J\right)^{-1} \cdot J^{T} \cdot z_{r e v}
$$

The damped least squares algorithm applies a damping factor in order to eliminate divergence. The performance metric is slightly changed:

$$
m_{\text {rev }}=\left\|z_{\text {rev }}\right\|=\left\|z-z_{\text {meas }}\right\|+p \cdot \boldsymbol{x}^{T} \boldsymbol{x}
$$

At each iteration, the following DLS calculation is done:

$$
\delta \boldsymbol{x}=-\left(\boldsymbol{J}^{T} \cdot \boldsymbol{J}+p \cdot \boldsymbol{I}\right)^{-1} \cdot \boldsymbol{J}^{T} \cdot \mathbf{z}_{r e v}
$$

Both for OD and DLS, the ray-tracing program solves the equation and through iterations calculates $\delta \boldsymbol{x}_{\text {final }}$.

\section{CASE STUDIES}

Phase diversity detailed in [1] and [2] provides the phase function (or WFE) in the pupil of the system. Using this information, misalignments can be corrected and the system's performance improved. In section 2, we investigated the coupling between the different degrees of freedom (DOF) of the system and their effect on the performance metric 
(WFE). The DOF, which are available for improving the instrument's performance are the compensators. Based on the type of compensator, two cases can be considered:

i. A system, where the basic adjustment of certain components is possible in the optical path, the performance metric could be compensated with decentres and tilts. In the most general case, each component has 6 degrees of freedom (DOF) for optimization: three offsets (decentres) and three rotations (tilts).

ii. A system, where the surface shape of a component (mirror) can be adjusted. Active mirrors are extremely useful for compensating residual fabrication errors or misalignments as well as to improve or adopt the performance of an instrument or component (using e.g. extreme aspherical mirrors).

In the following two sub-sections we investigate the mentioned two cases through a particular optical system. We selected to use the high-resolution spectrograph part of METIS to study the presented techniques. METIS is a proposed mid-infrared instrument for the E-ELT, which has a complex optical path based on a prism pre-disperser and an echelle based main disperser. The optical system of the main disperser is essentially a double pass Three Mirror Anastigmat (TMA), which include off-axis aspherical mirrors (Figure 1).

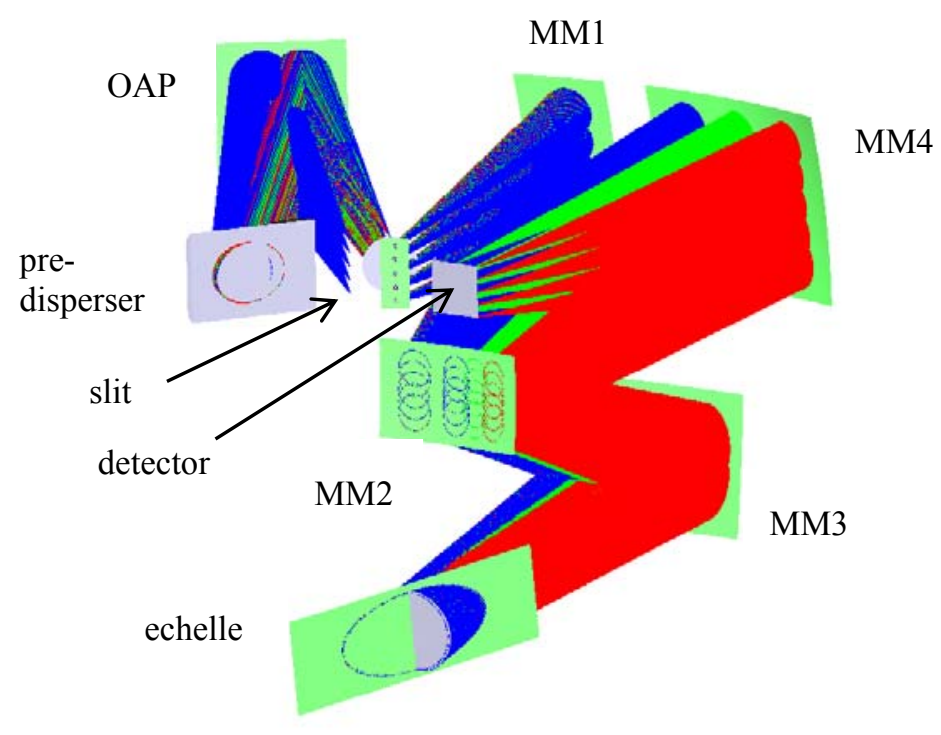

Figure 1 The layout of the METIS high-resolution spectrograph.

\subsection{Compensation based on tilt-displacement}

Tilt and/or displacement type compensation is necessary in order to correct WFE introduced by misalignments in the system. Through SVD of the sensitivity matrix of the METIS spectrograph, we investigate the orthogonal aberrations due to possible misalignments in the system. With the matrix regularization technique, we intend to find the best tilt and displacement type compensators. Finally we simulate random misalignments and using forward and reverse optimization methods we optimize compensator DOF.

For the calculation of the sensitivity matrix we take into account all relevant displacements (decentre $\mathrm{x}, \mathrm{y}$ and piston) and tilts (tilt along $\mathrm{x}, \mathrm{y}$ and $\mathrm{z}$ ), which result a configuration vector with 50 components. The aberration vector consists of the Zernike coefficients that represent image quality at three field positions (centre and the two edges of the slit) and three wavelengths $(4.616,4.65$ and $4.681 \mu \mathrm{m}$ ). We use 14 Standard (or Noll) Zernike coefficients, from z4 to z17, which means an aberration vector with an overall 126 components. The sensitivity matrix is a 126x50 matrix and the SVD results a $126 \times 126 \mathrm{U}$ matrix, 50x50 $\mathrm{V}$ matrix and 126x50 W matrix with the singular values at its diagonal. On Figure 2 the diagonal elements of $W$ are shown. As it was mentioned in section 2, the singular values of $W$ correspond to particular aberration modes. Essentially they represent the strength of each mode. On the following figure it can be seen 
that the first three modes are the most dominant in the case of the METIS spectrograph. The particular aberrations and the misalignments that caused these aberrations are shown in Table 1.

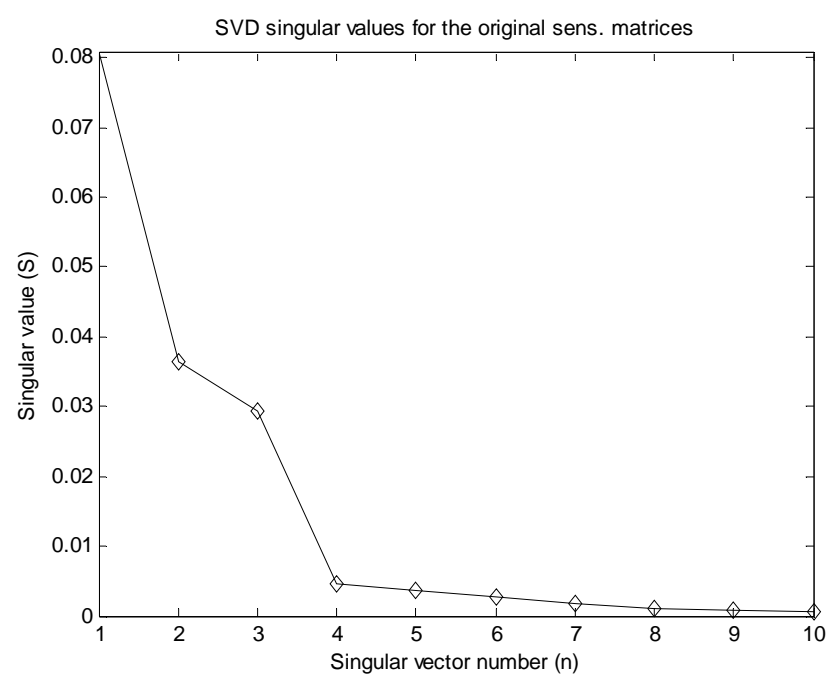

Figure 2 The singular values of $\mathrm{W}$ matrix.

Since the sensitivity matrix is formed from the aberration vectors caused by unit perturbations, the sensitivity matrix and the three matrices that result from the SVD depend on the defined units of perturbations. We used $50 \mu \mathrm{m}$ for displacements and 0.01 degree for tilts as unit perturbations to calculate the sensitivity matrix. These values are in the range of the achievable alignment tolerances of the system under investigation.

One way of representing the SVD of the sensitivity matrix is through the visualization of its matrices. The $\boldsymbol{U}$ and $\boldsymbol{V}$ matrices are illustrated in Table 1. Each mode is represented by the column vectors of $\boldsymbol{U}$ that show the type of aberrations present in that particular mode. The column vectors of $\boldsymbol{V}$ indicate the misalignments that caused these aberrations and they are measured in the units of the perturbations.

From the SVD the following can be obtained: $\boldsymbol{S} \boldsymbol{v}_{\boldsymbol{i}}=w_{i} \boldsymbol{u}_{\boldsymbol{i}}$. Each aberration and configuration singular vector $(\boldsymbol{U}$ and $\boldsymbol{V}$ column vectors) has unit norm and the singular values of $W$ have the same units as the sensitivity matrix, given aberration per unit perturbation. In the following Table the $\boldsymbol{U}$ aberration singular vectors are weighted with the singular values of $\boldsymbol{W}$ and the column vectors of $\boldsymbol{V}$ are measured in the units of the perturbations. 
Table 1 The most important modes with their specific aberrations $\left(2^{\text {nd }}\right.$ column$)$ and the misalignments that cause them ( $3^{\text {rd }}$ column) are shown. Misalignments are given in units of DOF adjustments (Y axis) as a function of DOF numbers $(\mathrm{X}$ axis).

\begin{tabular}{|c|c|c|c|}
\hline Modes & $\begin{array}{l}\text { Column of } \boldsymbol{U} \text { matrix } \\
\text { (aberrations for } 9 \quad \text { image } \\
\text { positions) }\end{array}$ & $\begin{array}{l}\text { Column of } \boldsymbol{V} \text { matrix } \\
\text { (misalignments of the } 50 \mathrm{DOF} \text { ) }\end{array}$ & Notes \\
\hline 1 & & $\hat{q}$ & $\begin{array}{l}\text { The first orthogonal } \\
\text { aberration mode consists } \\
\text { mostly of z4 (defocus) and } \\
\text { z6 (astigmatism) as a result } \\
\text { of piston and tilt of various } \\
\text { components. The largest } \\
\text { contributors are OAP and } \\
\text { MM3 regarding tilt y and } \\
\text { MM2 regarding piston. }\end{array}$ \\
\hline 2 & $\mathrm{R}$ & 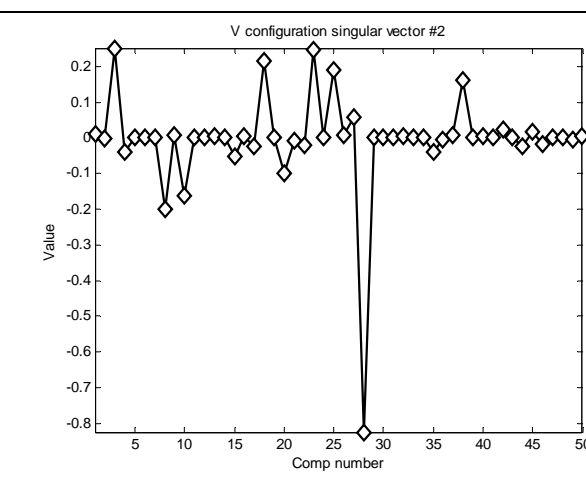 & $\begin{array}{l}\text { The second mode consists } \\
\text { primarily of z5 (astigmatism } \\
\mathrm{x} \text { ), and some minor } \mathrm{z} 4 \\
\text { (defocus) and z6 } \\
\text { (astigmatism y) are present. } \\
\text { This originates from a linear } \\
\text { combination of tilts of } \\
\text { certain components. Again } \\
\text { MM3 tilt y is the largest } \\
\text { contributor DOF. }\end{array}$ \\
\hline 3 & & 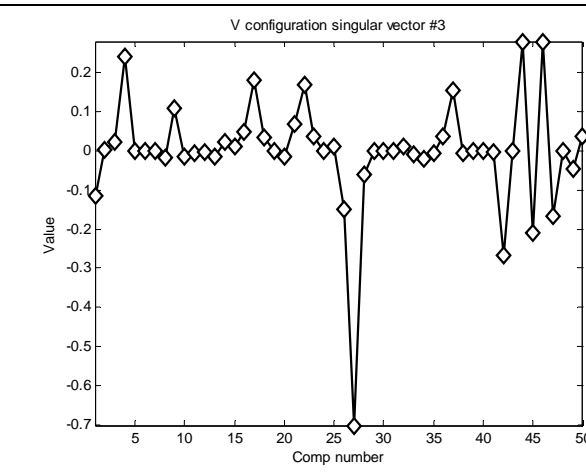 & $\begin{array}{l}\text { The third mode has mostly } \\
\text { z4 (defocus) and z6 } \\
\text { (astigmatism y) aberrations. } \\
\text { Largest contributors are } \\
\text { MM3 tilt } x \text { with piston type } \\
\text { misalignments of various } \\
\text { components. It is similar to } \\
\text { mode 1, but tilt x } \\
\text { misalignments are } \\
\text { contributors instead of tilt } y .\end{array}$ \\
\hline 4 & & 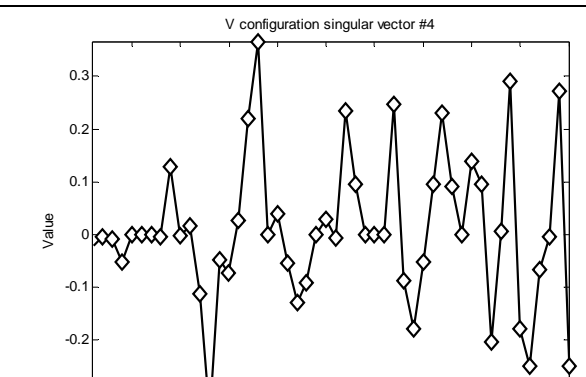 & $\begin{array}{l}\text { The fourth mode consists of } \\
\text { many aberrations ranging } \\
\text { from z4 to z12 mostly } \\
\text { dominated by astigmatism } \\
\text { and coma. It is originated } \\
\text { from a linear combination } \\
\text { of tilts and piston } \\
\text { movements of certain } \\
\text { components. }\end{array}$ \\
\hline
\end{tabular}


The regularization of the sensitivity matrix was done and it became apparent that the three most efficient compensator DOF are MM3 tilt x, tilt y and MM2 piston. Random misalignments were modelled and alignment runs were simulated with forward and reverse optimization. On Figure 3 WFE is shown as a function of optimization steps. The WFE is an average from 20 simulations (alignment runs) with random misalignments that result 0.09-0.10 waves RMS WFE (this is illustrated as iteration step 0). When only the three most sensitive compensators were used (MM3 tilt $\mathrm{x}$, y and MM2 piston) convergence could be achieved in all investigated cases and one iteration step was enough for finding the minimum. On the other hand, when less sensitive compensators were also used divergence was found in both optimization cases. Detector tilt $\mathrm{x}, \mathrm{y}$ and piston were added as compensators (6 DOF case on Figure 3) and additionally MM2 tilt $\mathrm{x}, \mathrm{y}$ and MM1 piston (9 DOF case on Figure 3) to include less sensitive DOF. The scenario with 9 compensator DOF resulted divergence (both reverse and forward optimization) as it can be clearly seen on Figure 3.

Although reverse optimization also provided convergence, the average WFE was always worse than in the forward optimization case and the standard deviation of the WFE was larger as well. E.g. in the 3 DOF case forward optimization always delivered a $0.033-0.037$ wave RMS WFE, while during reverse optimization it was varying between 0.035 and 0.075 waves. The nominal design RMS WFE, which is 0.035 waves was not achieved in the simulated cases.
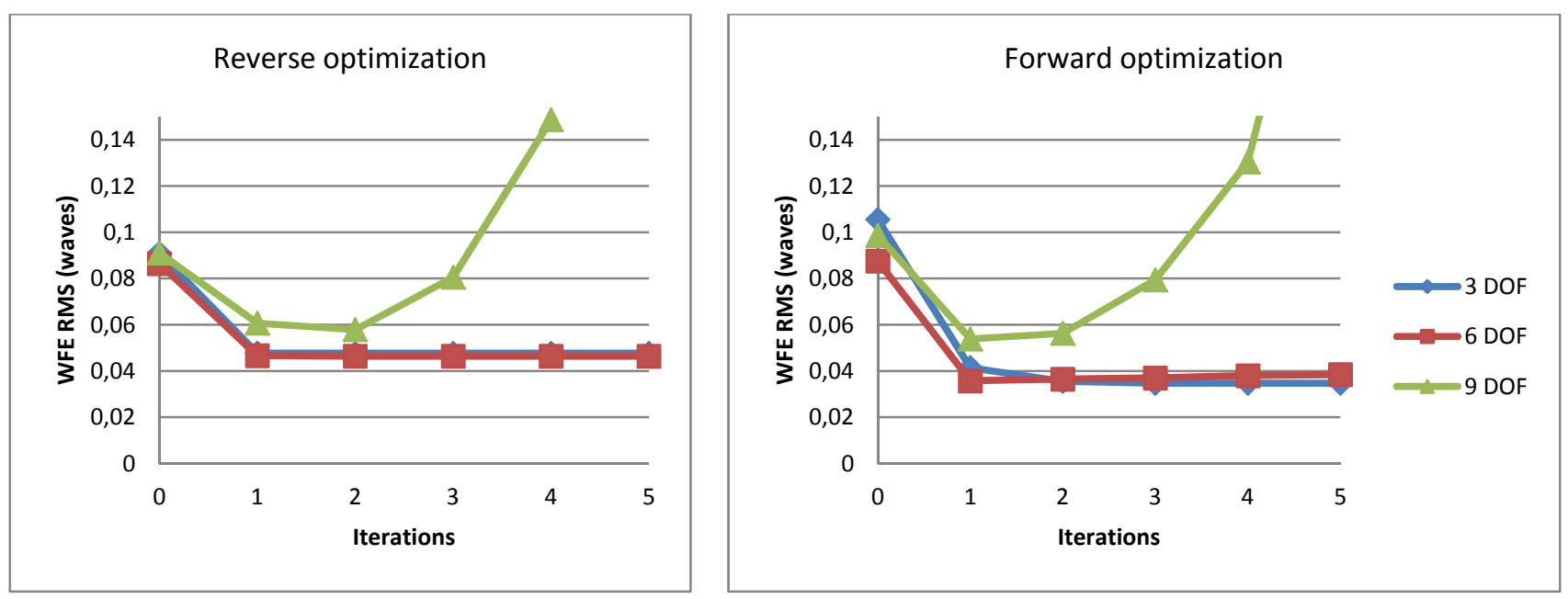

Figure 3 Performance metric (overall WFE) during the optimization (alignment) process. On the left when forward optimization is used with the Newton method, on the right, when reverse optimization is used. In all cases the WFE is the average from 20 random misalignments.

\subsection{Compensation based on surface deformation}

The wavefront information from phase diversity methods can be used directly to adjust a component with active surface, if it is located in the pupil. With the linear algebra techniques presented before, the correlation between the DOF of the active component and the performance metric can be found and the active component can be optimized even if it is not located in the pupil.

The same high-resolution spectrograph was used to model a component with active surface. MM3, the mirror closest to the pupil was transformed into an active component. The configuration vector components were defined as the adjustable DOF of the surface of MM3. For the ease of implementation the surface of MM3 was also characterized by Zernike polynomials, using the same 14 (z4-z17) Noll Zernike polynomials that were used for the characterization of the WFE. The aberration vector had 126 components composed from the wavefront at three field positions and three wavelengths. The sensitivity matrix and the SVD of the matrix were calculated similarly than before; the results can be seen on Figure 4 and in Table 2. 


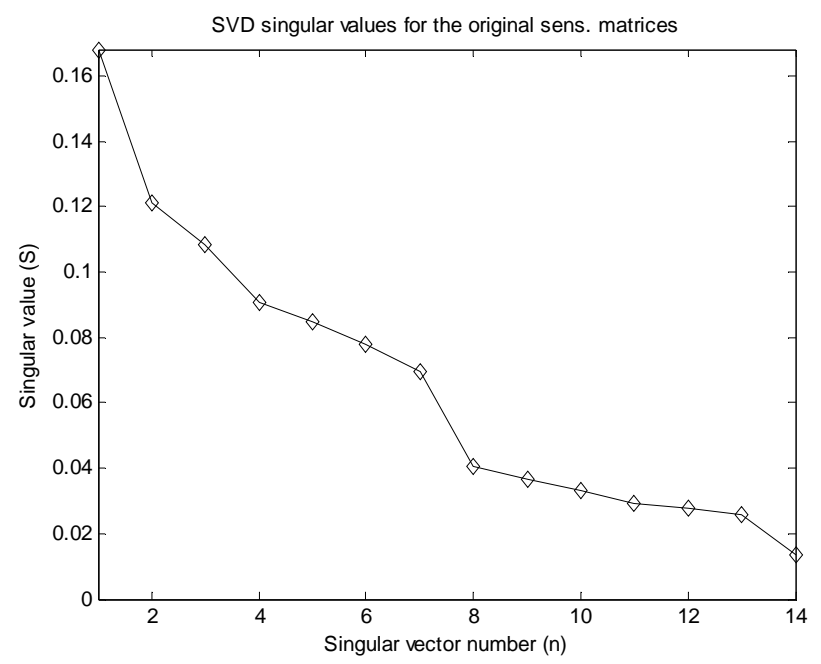

Figure 4 The singular values of the $\mathrm{W}$ matrix.

Table 2 The first six $\mathrm{V}$ configuration singular vectors are shown. It can be seen that the most dominant modes are results of the higher spatial frequency (comp number 8-14 that corresponds to z11-z17 Zernike terms) surface deformations of MM3.

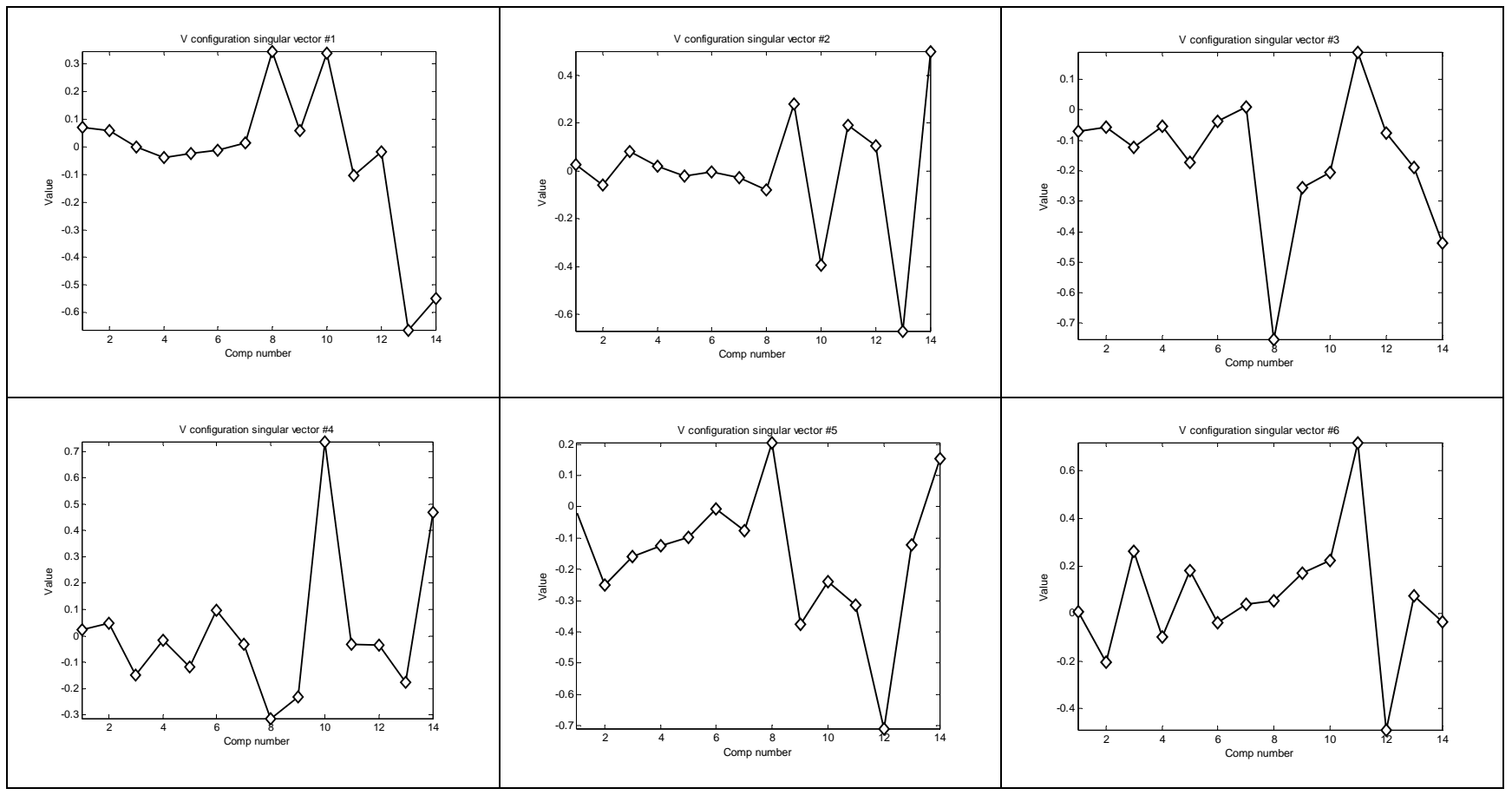


Random wavefronts resulting 0.05-0.06 waves RMS WFE were simulated and optimization of the active surface was done with forward and reverse optimization. The results can be seen in Figure 5. In all cases convergence can be seen. The plotted results correspond to average values, the RMS WFE is an average from 20 optimization runs. The nominal design RMS WFE is 0.035 . When the Zernike coefficients between $z 4$ and z17 were used to optimize the surface shape of MM3, the WFE correction could not reach the nominal configuration. On the other hand, when higher spatial frequencies were also optimized, the WFE could be reduced even below the nominal value (Figure 5 right).
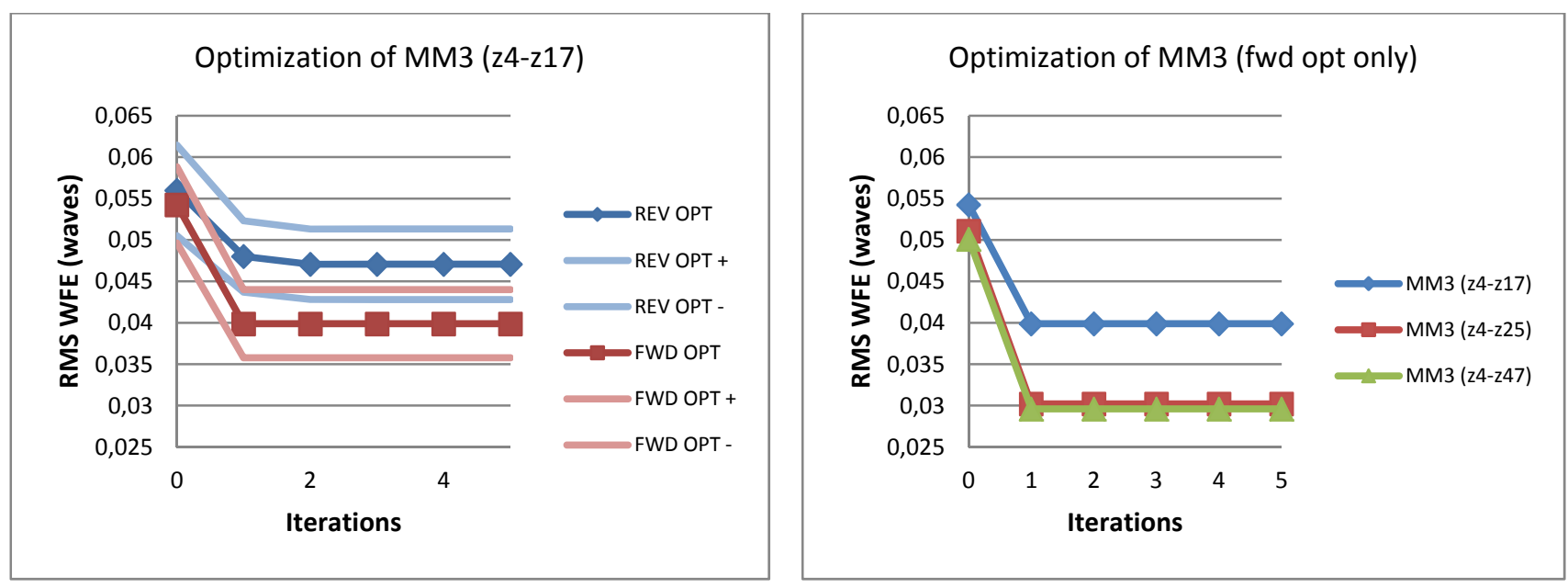

Figure 5 Performance metric (overall WFE) during the optimization process of MM3. In all cases the WFE is the average from 20 optimization runs. Left: forward and reverse optimizations are shown with standard deviation (+: standard deviation is added to the mean, -: standard deviation is subtracted from the mean). Right: different sets of Zernike polynomials are used to describe the surface shape of MM3. When higher spatial frequencies are also included, the RMS WFE improves, even further than the nominal RMS WFE (0.035 wave).

The optimization algorithms were tested successfully and they showed convergence during the optimization of the surface of MM3. In order to test the algorithms in a less ideal situation, the surface optimization of MM4 was also investigated. As opposed to MM3, which is located close to the pupil, MM4 is the last optical component in the optical path, positioned close to the focal plane, just before the detector. The same analysis was done as for MM3; the results are shown in Figure 6. It became apparent that it is even more important to increase the adjustable spatial frequencies on the surface of MM4. When z4-z17 polynomials were optimized, hardly any WFE improvement could be seen. When higher spatial frequencies were also included in the optimization process, the WFE could be further minimized.
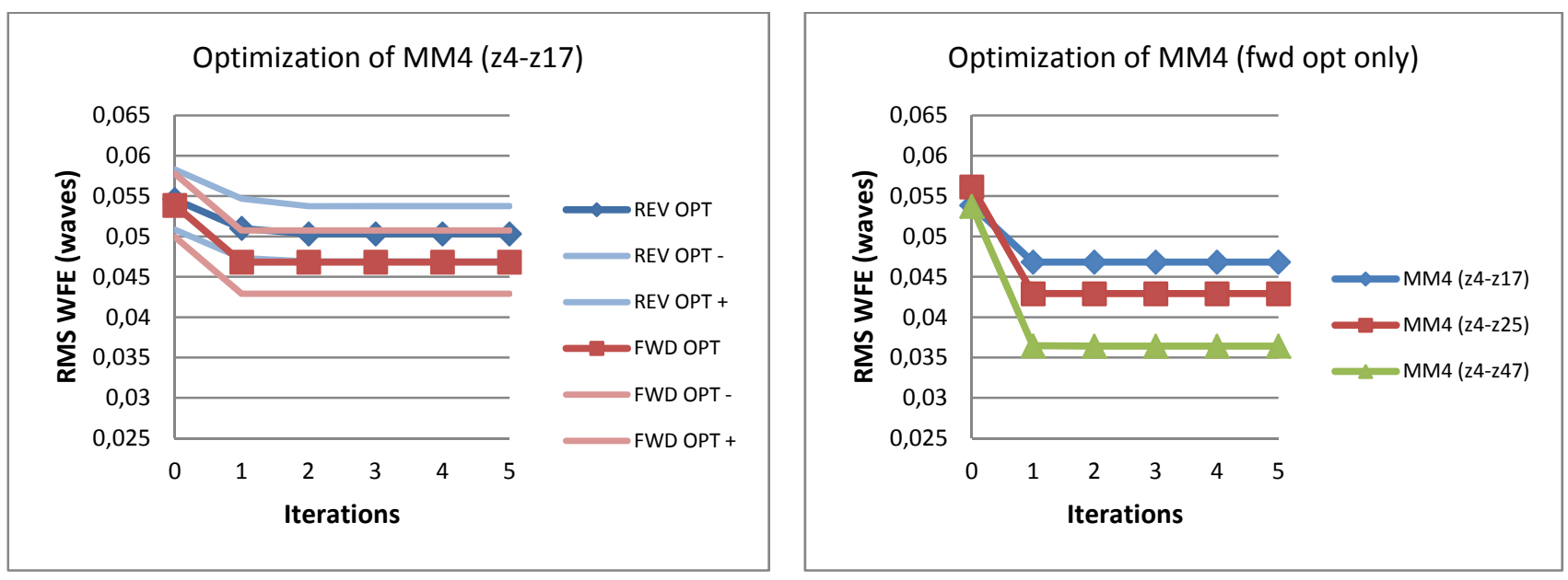

Figure 6 Same as Figure 5, but the optimization of MM4 is shown. In the case of MM4 the higher frequency Zernike polynomials (that control of the surface of MM4) are even more important than in the case of MM3. 
Presumably the reason for the inevitability of the high spatial frequency coefficients to reduce WFE is that MM4 is the last optical component before the detector, which means that the footprint of the beam is smaller and consequently smaller scales of the mirror should be controlled in order to reduce aberrations.

\section{CONCLUSIONS AND THE NEXT PHASE}

We studied SVD and regularization of the sensitivity matrix and investigated forward and reverse optimization methods that can be used to optimize the active optical component.

First we investigated a system, where basic adjustment of certain components is possible in the optical train. The WFE could be compensated with decentres and tilts. We came to the conclusion that as long as insensitive DOF are not included as compensators, both reverse and forward optimization methods can be used efficiently. When insensitive DOF are used, the optimization processes start to diverge. We tested our algorithms with a double pass TMA based spectrograph, selected the best set of compensators in the system, simulated randomly misaligned systems and optimized the selected compensators in order to minimize the selected performance metric, the WFE.

We also studied a system, where the surface shape of a component can be adjusted and the component not necessarily has to be located in the pupil. The same double pass TMA based spectrograph was used as a test-system and first the surface deformations of a mirror close to the pupil were optimized in order to minimize WFE. The algorithms were also tested in a less ideal situation, where the component with adjustable surface deformations is positioned close to the focal plane, just before the detector. We simulated random wavefronts to model misalignments and polishing errors and successfully optimized the surface deformations of the active component.

Comparing forward and reverse optimization, it became apparent that each method has its own advantages and disadvantages and in every particular situation it needs to be judged which is the more efficient one to use. Since the optimization process is controlled through a programming language, forward optimization is more flexible, e.g. it is easier to implement mathematical constraints. Another advantage is that the sensitivity matrix can be determined experimentally, so the linearization is at the point of interest, which means that a more accurate sensitivity matrix can be calculated and better performance achieved. Reverse optimization uses a ray-tracing program to find the best adjustments of the selected compensator DOF. The sensitivity matrix is calculated in the background by the embedded optimization algorithms, so it is difficult to get information on the eigenvectors of the system. Nevertheless it is relatively easy to implement reverse optimization with a ray-tracing program and in many cases it is sufficient to arrive to the necessary compensator adjustments.

In the next phase we intend to connect phase diversity with the optimization process and perform end-to-end simulations. Phase diversity will be used on one hand to measure the aberration vector and create the sensitivity matrix, on the other hand to measure wavefront aberrations due to simulated misalignment and surface shape errors. Phase diversity and Gerchberg-Saxton algorithms for active systems are investigated by Korkiakoski et al in [1] and [2] and we intend to use the outcome of these studies in order to build a complete simulation tool that can be used to model more realistic scenarios. We also plan to build a prototype system, which can be used to test our developed phase retrieval and optimization methods.

\section{ACKNOWLEDGEMENTS}

"This research, part of the OPTICON program, is supported by the European Commission's FP7 Capacities programme (Grant number 226604)."

\section{REFERENCES}

[1] Korkiakoski, V., A., Venema, L., Agocs, T., Keller, C., U., Doelman, N., Fraanje, R., Andrei, R., Verhaegen, M., "Potential of phase-diversity for metrology of active instruments," Proc. SPIE 8450, 200 (2012).

[2] Korkiakoski, V., Keller, C., U., Doelman, N., Fraanje, R., and Verhaegen, M., "Joint optimization of phase diversity and adaptive optics: demonstration of potential," Appl. Opt. 51, 102-113 (2012). 
[3] Chapman, H., N., Sweeney, D., W., "A rigorous method for compensation selection and alignment of microlithographic optical systems," Proc. SPIE 3331, 102-13 (1998).

[4] Hvisc, A., M., Burge, J., H., "Alignment analysis of four-mirror spherical aberration correctors," Proc. SPIE 7018, 19 (2008).

[5] Golub, G., H., Van Loan, C., F., "Matrix Computations," John Hopkins University Press, Baltimore, $3^{\text {rd }}$ ed. (1996).

[6] Griffith, D., "How to Talk to Zemax from MatLab"

http://www.radiantzemax.com/kb-en/KnowledgebaseArticle50204.aspx 